\title{
Two-Level Authentication System for Access Gate Entry in a Localized Campus Network
}

\author{
Kartik Joshi, Tushar Nama, Rahul Hemal Shah, P. Vijayakumar
}

\begin{abstract}
Managing the people in a vast organization is a crucial and challenging task that needs to be simple and efficient to be implemented in an organization with a large workforce. Authentication System technology has evolved over the years from the traditional manual verification to biometric technology. These approaches at some point of time have raised concern over the time consumption, range and bandwidth issues. An efficient solution for employee management through authentication is designed using the ESP8266 Wi-Fi module present in NodeMCU microcontroller. Employees connected to the Wi-Fi network of the organization could be tracked and the authentication process could be automated using the IP web camera that extracts the number plate of the vehicle. The vehicle Number plate is detected using a Web camera. This is done by the Computer Vision technique that extracts the region of the image based on the gradient difference found in the number plate. Access gate entry and exit are automated using database information, thus avoiding manual labour work. For a big organization, this could be more efficient than RFID Technology as this design helps the employee avoid the hassle of manually giving the attendance.
\end{abstract}

Keywords: Access Gate Authentication, Web camera, Number Plate Recognition, Automobile Mapping, Wi-Fi ESP8266.

\section{INTRODUCTION}

With the advent of globalization and the Industrial Revolution, there are many industries that are coming up. With an increasing number of industries, there is a severe need of employee management system for the organization to work productively and keep track of their employees. Authentication Management System for employee access is a great way to keep track of all the employees of the

Revised Manuscript Received on February 05, 2020.

* Correspondence Author

Kartik Joshi, B.Tech. (Electronics and Computer Engineering) III Year Student, School of Electronics Engineering, Vellore Institute of Technology (VIT) Chennai, Tamil Nadu 600127, India. Email: kartik.joshi2017@vitstudent.ac.in

Tushar Nama, B.Tech. (Electronics and Computer Engineering) III Year Student, School of Electronics Engineering, Vellore Institute of Technology (VIT) Chennai, Tamil Nadu 600127, India. Email: tushar.nama2017@vitstudent.ac.in

Rahul Hemal Shah, B.Tech. (Electronics and Computer Engineering) III Year Student, School of Electronics Engineering, Vellore Institute of Technology (VIT) Chennai, Tamil Nadu 600127, India. Email: rahul.shah2017@vitstudent.ac.in

Dr. P. Vijayakumar, Associate Professor, Vellore Institute of Technology, Chennai, Tamil Nadu 600127, India. Email: vijaya.kumar@vit.ac.in

(c) The Authors. Published by Blue Eyes Intelligence Engineering and Sciences Publication (BEIESP). This is an open access article under the CC BY-NC-ND license (http://creativecommons.org/licenses/by-nc-nd/4.0/) organization. Access Gate Authentication also helps the companies track the commitment of the employees and in reorganizing the company structure through credit-based employee promotions based on attendance as one of the metrics. Current Access Gate Authentication System varies depending upon the scale of operation of the organization. Small scale industries prefer manual authentication. Medium Scale companies tend to prefer the RFID based authentication system. At present, organizations at large scale have implemented biometric based access gate authentication system. The Limitation with the current systems includes execution time, security and privacy concerns.

We propose a novel approach of Authentication System for Employee Access through IP mapping of the employee owned IoT device such as mobile headsets, smartwatches, etc. with the automobile's number plate. Employee of the company is given access to the campus by verifying the number plate with the Employee's IP that corresponds to the organizational database. Upon successful verification, the employee details are registered in the database with the corresponding time stamp for future reference. The proposed system essentially aims at reducing the execution time of the Access Gate Authentication without compromising on the performance of the system. Contribution of the Author: Our work deals with the Automobile - IP mapping for automatic smart attendance system that authenticates the employee privileges and provides appropriate access to the employee. The walk-in access gate authentication system proposes is to store IP Address, Vehicle Number Plate in the organizational database that is responsible for authentication of the employee.

This paper is well organized as follows: current chapter introduces about attendance management system for access gate authentication and its limitation along with the contribution of the authors. Section II gives the related work about the attendance management system. Section III briefly describes about the conventional attendance management system along with its limitation. Section IV proposes the Wi-Fi based Access gate entry System. Section V shows the implemented results. Finally, paper concludes with a mention on the scope of the future work towards this system.

\section{RELATED WORKS}

Jagdish [1] describes the entry management system using the RFID tag entry system and mailing the list using the SMTP server. But the limitation is a person should be at a max $10 \mathrm{~cm}$ distance from the reader because a general RFID reader has a normal range of $10-12 \mathrm{~cm}$. 


\section{Two-Level Authentication System for Access Gate Entry in a Localized Campus Network}

Then they are sending the data to the manager which extracts the data from the database. Lia[2] describes the Bluetooth based entry system using an android app and Arduino. They have developed an android app that uses the device Bluetooth to contact the Arduino and give the command of the opening of the door. But the limitation is that a person has to carry that particular device which makes it a little difficult to implement in public places and this system is very easy to be hacked.

Kadry [3] goes on to explore the authentication domain with Iris recognition. While Iris remains, the unique characteristics associated with a person. Calibration and Verification of such system compromises the tradeoff between performance and execution time. Such limitation prevents efficient access gate entry. SriKanth[4] describes the Biometric based gate entry system using the fingerprint sensor, Raspberry Pi and cloud platform for checking the entry so that no outsider can get into the campus and using IoT to make the system secured and compatible. But the limitation is that a person has to get out of the vehicle and wait in the queue to give the biometric which is time-consuming and may lead to entry blockage. Kar [5] proposed a system design with an approach of integrating face recognition module. The results found where accurate with a slight compromise on the execution time.

\section{TRADITIONAL ATTENDANCE MANAGEMENT SYSTEM}

Traditional manual entry method for Gate Access Gate Entry System was found to be very time consuming as well as responsible for manual error. To reduce the error, Radio Frequency Identification (RFID) based Gate Access Entry system came into existence. While it could efficiently provide employee information, it had a major setback of portability. RFID Tags could be easily exchanged, so it was not reliable. RFID Technology was followed by Biometric Systems that used finger print for authentication. This was able to account for reliability. One major concern about the Biometric System was related with the dynamic employee tracking and the person has to either get out from the vehicle to put the biometric or wait in the queue. Employees working could not be accessed when needed in case of emergency. With the advent of computational power, Convolutional Neural Networks became feasible to be deployed in real time authentication systems. However, they still suffer from a setback at the deployment stage pertaining to the execution time.

\section{PROPOSED TWO LEVEL AUTHENTICATION SYSTEM FOR ACCESS GATE ENTRY SYSTEM}

This system presents a novel approach for Access gate entry System. In an industry spread over a large area, its network could be used effectively for authenticating the information of employees and their vehicle number so that any outsider could not enter easily. We can also implement this to track the location of the employee in the campus area. Access gate entry could be automated using a web camera setup with the OpenCV model. NodeMCU has on-board Wi-Fi module ESP8266, that could be used for the network. We installed a WiFi chip on the id card of user so that it can connect to Node-MCU and this chip is cheap and compatible and easy to attach to id card. Web camera is used to send the number plate image to the central system. Central System uses Image processing technique using OpenCV library to identify the employee from its database. Upon identification, employee gets the access to enter the area and attendance can also be recorded from the same.

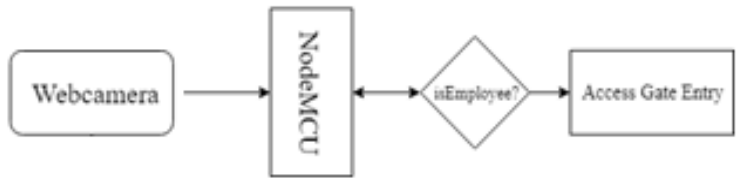

Fig. 1. Block diagram for automatic attendance system in a localized campus network.

IP Address, MAC Address, Vehicle number plate image and other personal details are stored in the database of the organization. When the employee gets connected to the campus network, the IP Address, MAC Address, Vehicle Number Plate image, Employee ID, and other personal details is matched with the one present in the database. If the details match with the database profile, access gate entry is given to the employee and the Attendance is marked for that employee.

\section{A. Wi-Fi Entry Gate using ESP8266}

ESP8266 is a Wi-Fi module with full stack TCP/IP. Once the device is connected to the Wi-Fi module, MAC Address and IP Address is listed in the serial monitor. This is matched with the database. After successful authentication, access gate opens automatically, marking the attendance. In case the employee details do not match with the existing database, buzzer alerts the administrator.

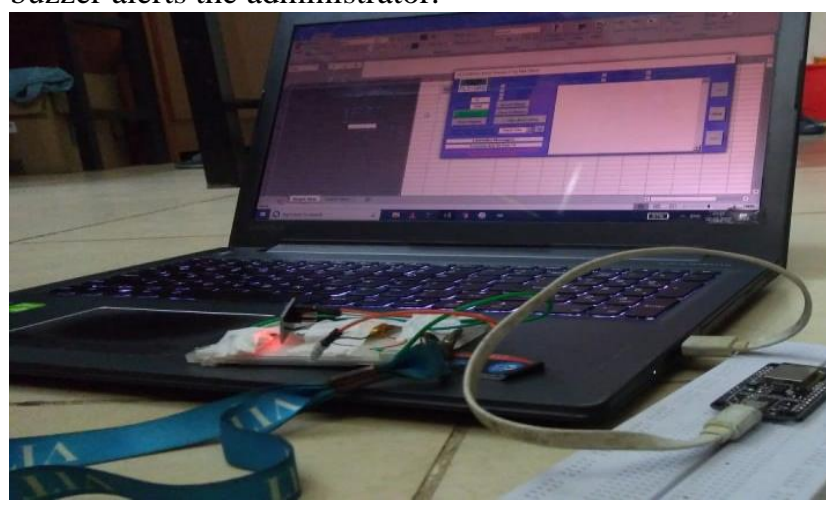

Fig. 2. Model of the Access Gate Entry System.

Fig 2 shows the configuration of the Access Gate Entry system. The Node-Mcu is connected to a laptop that acts as the main server and the id card configured with the Wi-Fi module act as a user. Every Wi-Fi module has been configured with a unique IP address and MAC address so that it can differentiate among the user and this setup is done using the AT commands through serial monitor and Wi-Fi module connected to the Arduino device. Upon connection to the campus network, the central system can know about the connected device as the MAC Address, IP Address of the connected device is known. The values of the MAC address and IP Address can be passed to the database for querying and retrieval of the employee details. 
Number Plate image is sent to the central server and the number plate is extracted. This is matched with the pre-existing number plates and access gate entry is authenticated accordingly.

\section{B. Software Interface}

ARDUINO IDE- Arduino Integrated Development Environment is an application software that is written in $\mathrm{C} / \mathrm{C}++$. It is used to write and upload the programs for the Arduino compatible boards. We are using it for connecting the Wi-Fi device to server and sending the data to the database.

PLX-DAQ - PLX-DAQ is a simple add-on software for Excel that makes it easy to create the database by taking the input from the Node-MCU same as the serial monitor.

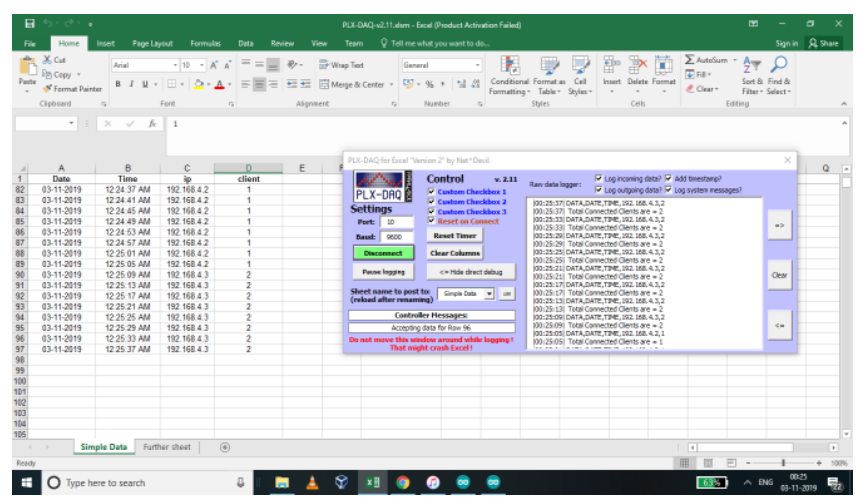

Fig.3. Database Software taking the real time entry

TABLE 1 - Sample Output for IP and clients connected

\begin{tabular}{|l|l|}
\hline IP ADDRESS & CLIENTS CONNECTED \\
\hline 192.168 .1 .3 & 1 \\
\hline 192.168 .2 .5 & 2 \\
\hline 192.168 .3 .6 & 3 \\
\hline 192.168 .4 .5 & 4 \\
\hline 192.168 .1 .1 & 5 \\
\hline
\end{tabular}

This is a midway to connect the Arduino serial port to Microsoft Excel which is used to store the data of the devices connected to network through Node-Mcu. From this, we can extract the data and check the entry for the visitors and can be clubbed with their vehicle information which we are monitoring through openCV. This real-time database is easy to create and monitor that's why it can be used by even those who don't have any knowledge of microcontroller or database. Fig 3 shows the overview of the PLX-DAQ software which we are using it for Access Gate Entry System and also the real time input data along with date and time.

For setting up the PLX-DAQ according to one's need we can mention the name of columns with a keyword 'label' separated by comma and output with the keyword 'data' in the Arduino Ide. There is no need for importing any library for configuring the PLX-DAQ. It is possible to generate real - time graphs.

IMAGE PROCESSING USING OpenCV - For number plate recognition, the image is converted to grayscale and then we obtain intended region of interest through contour segregation. We use YOLO architecture [7] for Car Detection.
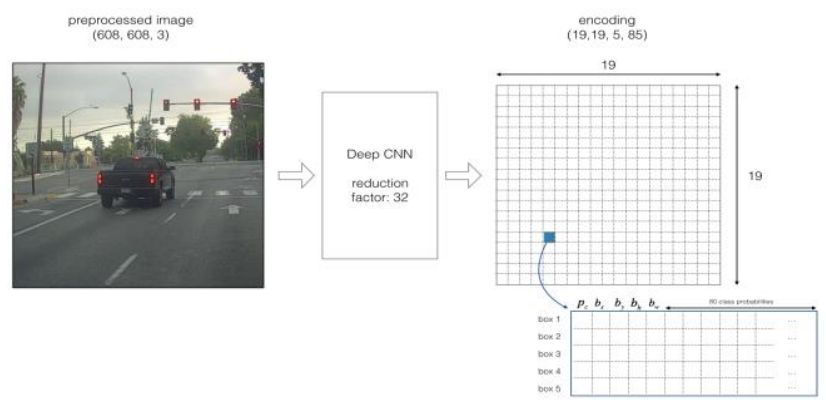

Fig. 4. Encoding Architecture for YOLO [7]

The probability classes of the region are defined by the anchor box. And the overlapping of the anchor boxes is reduced using Non-Max Suppression. The probability of anchor class to detect a particular object depends on the threshold score. Non-Max suppression is implemented by the IoU metric that considers the Intersection and Union of the anchor boxes in the desired region of interest.
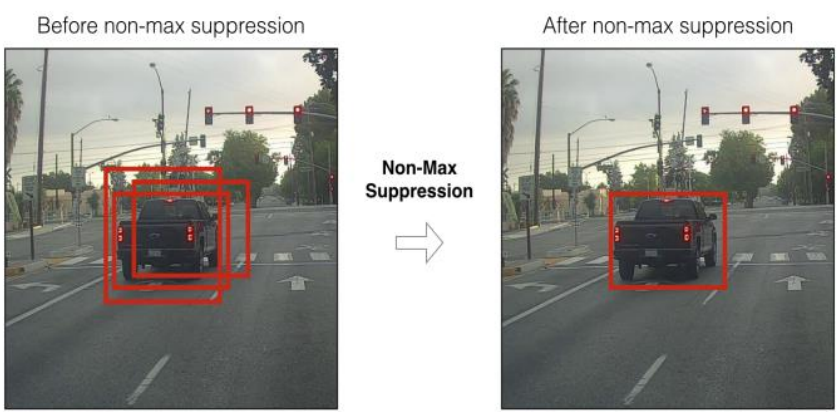

Fig. 5. Non-Max Suppression over the Drive.ai sample dataset [10]

After the stage of Car Detection, Number Plate of the automobile is extracted using OpenCV techniques as proposed by Anuj [8].

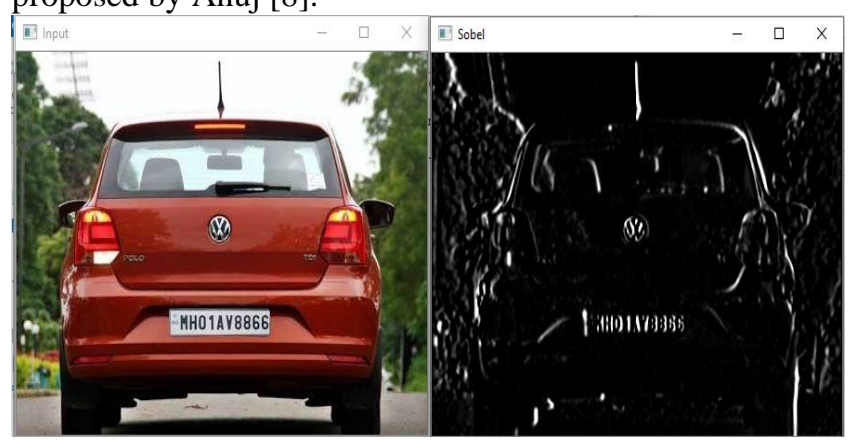

Fig. 6. Contour Extraction using OpenCV.

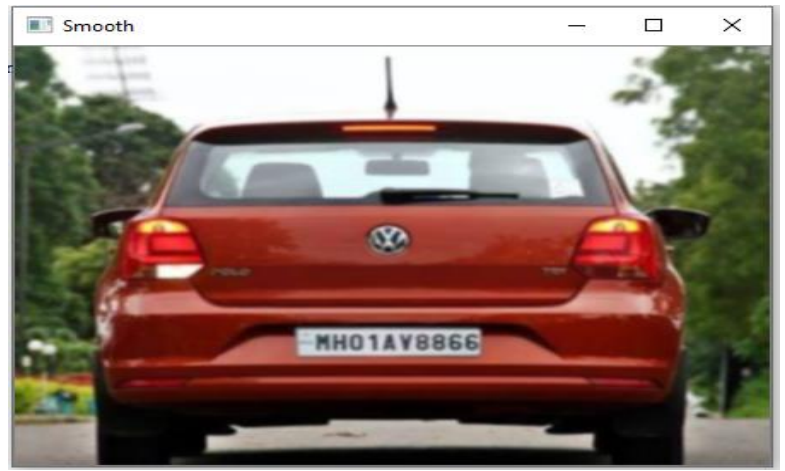

Fig. 7. Smoothing using Otsu Binarization in OpenCV. 


\section{- Cleaned Plate}

\section{HHO1AYGB6G}

Fig. 8. Extracted image of the Number Plate.

\section{Hardware Interface}

1. ESP8266 - ESP-8266 is low cost Wi-Fi chip used to establish a network that helps to connect it to other devices. "This module is the most famous one, as it is really small and only costs around ₹ 150 . However, the number of accessible GPIO pins (input/output pins) is quite limited. It is also difficult to plug it into a standard breadboard. The most basic way to use the ESP8266 module is to use serial commands, as the chip is basically a Wi-Fi/Serial transceiver" [9]

This has been attached with the ID card after setting the IP and MAC address of a device using the Arduino and AT commands. This is powered using $3.3 \mathrm{~V}$ lithium battery which doesn't harm the device. If even $5 \mathrm{~V}$ power is supplied to it then there are chances that it may get damaged. This act as a user device to the system and the information can be matched by the server for authentication.

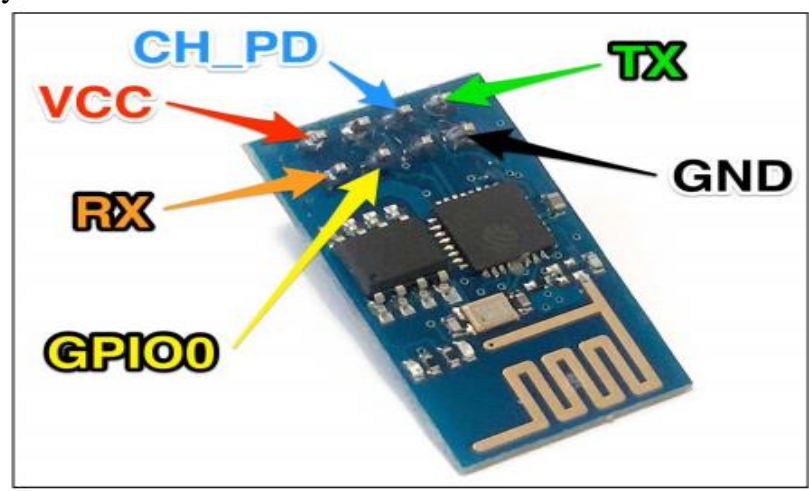

Fig. 9. ESP8266 with pin explanation [9]

2. NodeMCU - NodeMcu is a development kit which is already installed with ESP8266 module mainly used for IoT Platforms. In Access Gate Entry System Node-Mcu act as a server which connects the clients and store the IP and MAC address to validate the entry and it also receives the information from the OpenCV that the vehicle number matches with the specified person owning the vehicle.

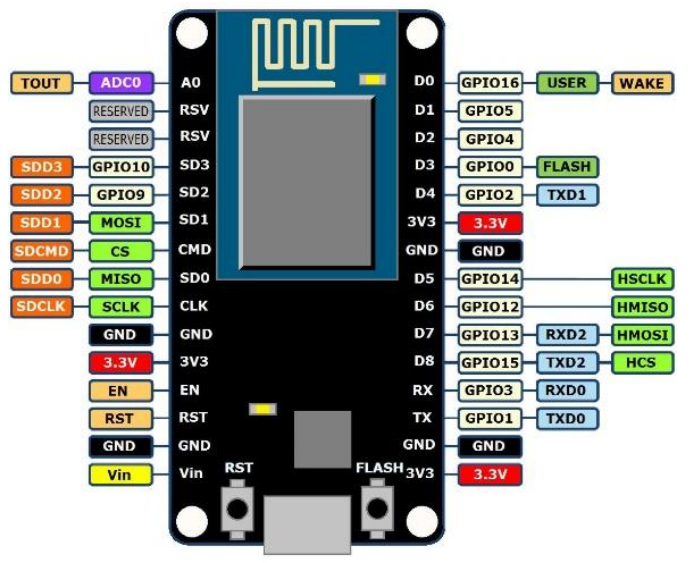

Fig. 10 Nodemcu with pin diagram

Fig 10 shows the pin diagram of the Node-Mcu and the structure. We can see that it is installed with ESP8266 module at the center. We also use Nodemcu or Arduino to setup our ESP8266 module using AT commands and serial monitor. So that whenever NodeMcu finds the IP of the module it tries to make a connection between them and get the data from the esp8266 and transfer it to plx database.

\section{IMPLEMENTATION RESULTS}

This section shows the results of the entire project, the result is printed on the excel sheet and we use databases to log entries. So, when a vehicle passes by the gate its NodeMCU connects and communicated with the NodeMCU kept at the gate and the IP of the client NodeMCU is received and the matched details are printed in excel. Thus, we can imply that the entry's shown in the excel sheet reveal the identity of the registered individual passing through the gate. Also, another implementation result will be the image processing of the vehicle plate number. Which helps us know that only registered and authorized vehicle numbers are granted access. This acts as a 2-step verification whereby when the registered individual passes through the gates and the camera detects the number plate of the vehicle and if it matches with the entry in the database then only the vehicle is granted access into the campus.

\section{CONCLUSION}

Thus, in this section we would like to conclude that the method of Two-level Authentication System for Access Gate Entry in a Localized Campus Network provides utmost security and also eliminates the need of guards at the gate. The technology used is very responsive and doesn't waste time compared to other conventional methods. Also, we use 2 level verification system which makes the entire model extremely reliable and robust. The entire model is designed and implemented based on the ideology of being quick, and cost efficient. We also are providing a solution to the real-world problem of unauthorized vehicle being granted access which is faced by almost all universities and office campus. We also have provided a solution where very minimal or no manual intervention is required and thereby promoting the use of technology in everyday life. This model also helps companies to move towards industry 4.0 by allowing full automation derived gate entry and exit.

\section{REFERENCES}

1. Jagdish Lal Raheja, Shashikant Nayak and Ashutosh Gupta," RFID BASED NETWORKED GATE ENTRY CONTROL SYSTEM (GECS)", International Journal of Computer Networks \& Communications (IJCNC), Vol.1, No.3, October 2009

2. Lia Kamelia, Alfin Noorhassan S.R, Mada Sanjaya and W.S., Edi Mulyana," DOOR-AUTOMATION SYSTEM USING BLUETOOTH-BASED ANDROID FOR MOBILE PHONE", ARPN Journal of Engineering and Applied Sciences, VOL. 9, NO. 10, OCTOBER 2014

3. Kadry, Seifedine, and Mohamad Smaili. "Wireless attendance management system based on iris recognition." Scientific Research and essays 5.12 (2010): 1428-1435.

4. SriKanth Murikipudi and K.HariPriya." Biometric Based Gate Access and Informative System through Web Services and SMS Using IOT", International Journal of Pure and Applied Mathematics, Volume 118 No. 20 2018, 2759-2774 
5. Kar, Nirmalya, et al. "Study of implementing automated attendance system using face recognition technique." International Journal of computer and communication engineering 1.2 (2012): 100.

6. Ip and Mac retrieval of the connected devices using serial terminal commands,

https://circuits4you.com/2018/01/31/esp8266-get-ip-mac-addr ess-of-connected-devices/amp/

7. You Only Look Once, Unified, Real-Time Object Detection, arXiv:1506.02640

8. Indian Number Plate Recognition System, 2018, Anuj Badhwar, https://github.com/anuj-badhwar/Indian-Number-Plate-Recog nition-SystemConference Short Name:WOODSTOCK'18

9. Marco Schwartz,"Internet of Things with ESP8266", Packt Publishing,2016

10. Drive.ai by Andrew Ng Research Lab is licensed under Creative Commons Attribution 4.0 International License.

11. Contour Extraction and Otsu Binarization using OpenCV, https://docs.opencv.org/

\section{AUTHORS PROFILE}

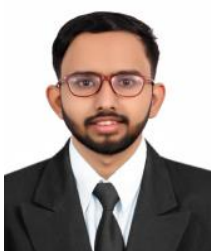

Kartik Joshi is studying third year Bachelor of Technology (Electronics and Computer Engineering) at Vellore Institute of Technology (VIT) Chennai, India. $\mathrm{He}$ is interested in learning new emerging technologies. His research areas of interest also include Machine Learning, Computer Vision, Natural Language Processing.

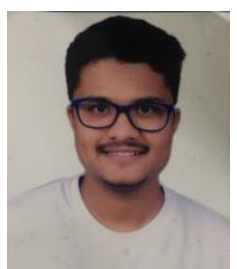

Tushar Nama is studying third year Bachelor of Technology (Electronics and Computer Engineering) at Vellore Institute of Technology (VIT), Chennai, India. He is interested in doing research work on different technologies and innovating new. His interest is in fields of Machine Learning, Artificial Intelligence and DevOps

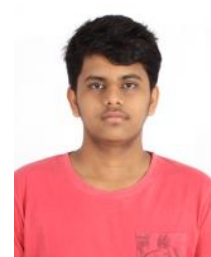

Rahul Hemal Shah is pursuing his third year of Bachelor of Technology in Electronics and Computer Engineering at Vellore Institute of Technology (VIT Chennai). He exhibits immense interest in research work through innovative ideas and generating optimum solutions. He is also interested in the field of artificial intelligence, computer vision, machine learning and embedded system design. He has done several innovative projects using computer vision, machine learning, Arduino, ESP8266, and many other microcontrollers. He is also versatile in coding languages such as $\mathrm{C}, \mathrm{C}++$, python, assembly level language.

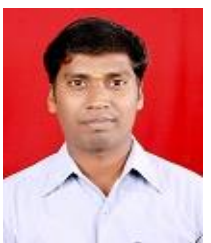

Dr. P. Vijayakumar is currently working as Associate Professor in School of Electronics Engineering at VIT University Chennai campus, India and completed his $\mathrm{PhD}$ in Wireless Security at Pondicherry University during 2015. He has totally 12 years of teaching and research experience and published more than 40 research papers in SCOPUS /SCI Indexed National / International Journals and Conferences. His area of specialization is Elliptic and Hyperelliptic Curve Cryptography, Blockchain technology, Cryptography and Network Security, Cryptographic Algorithms, DNA Steganography, Embedded System and IoT. 